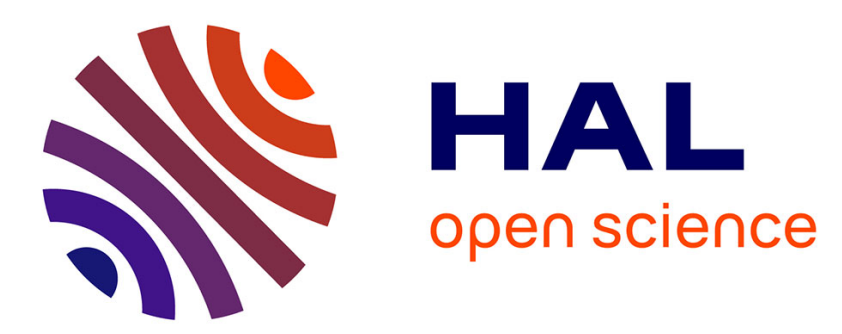

\title{
Noise of an overexpanded Mach 3.3 jet: non-linear propagation effects and correlations with flow
}

Nicolas de Cacqueray, Christophe Bogey

\section{To cite this version:}

Nicolas de Cacqueray, Christophe Bogey. Noise of an overexpanded Mach 3.3 jet: non-linear propagation effects and correlations with flow. International Journal of Aeroacoustics, 2014, 13 (7), pp.607-632. 10.1260/1475-472X.13.7-8.607 . hal-01136599

\section{HAL Id: hal-01136599 \\ https://hal.science/hal-01136599}

Submitted on 11 Apr 2016

HAL is a multi-disciplinary open access archive for the deposit and dissemination of scientific research documents, whether they are published or not. The documents may come from teaching and research institutions in France or abroad, or from public or private research centers.
L'archive ouverte pluridisciplinaire HAL, est destinée au dépôt et à la diffusion de documents scientifiques de niveau recherche, publiés ou non, émanant des établissements d'enseignement et de recherche français ou étrangers, des laboratoires publics ou privés. 


\title{
Noise of an overexpanded Mach 3.3 jet: non-linear propagation effects and correlations with flow
}

\author{
Nicolas de Cacqueray* and Christophe Bogey ${ }^{\dagger}$ \\ Laboratoire de Mécanique des Fluides et d'Acoustique, UMR CNRS 5509 \\ Ecole Centrale de Lyon, Université de Lyon, 69134 Ecully, France
}

Received: May 12, 2014; Revised: Oct. 28, 2014; Accepted: Nov. 1, 2014

\begin{abstract}
The noise emitted by an overexpanded round jet at a Mach number of 3.3 and a Reynolds number of $10^{5}$, computed in a previous study using large-eddy simulation (LES), is investigated. In a first step, the non-linear sound propagation effects are quantified by performing two far-field wave extrapolations from the LES near-field data. The extrapolations are carried out by solving the linearized Euler equations in one case and the full Euler equations in the other, without atmospheric absorption, up to a distance of 240 radii from the jet nozzle exit. The non-linear effects are shown to be quite significant, resulting in a series of $N$-shaped waves in the pressure signals, and in weaker mid-frequency components and stronger high-frequency components in the spectra. Close to the peak directivity radiation angle, for instance, they lead to about a $8 \mathrm{~dB}$ loss and a $6 \mathrm{~dB}$ gain at the Strouhal numbers of 0.2 and 1, respectively. In a second step, noise generation mechanisms are discussed by calculating correlations between far-field pressure fluctuations and turbulent quantities in the jet. High levels of correlation are found with the centerline flow fluctuations at the end of the potential core, with the shear-layer flow fluctuations over a large axial distance, and with the centerline density fluctuations between the $3 \mathrm{rd}$ and the 5th shock cells. They are attributed to the intermittent intrusion of low-speed vortical structures in the potential core, to the supersonic convection of turbulent structures, and to the shock motions at the screech tone frequency.
\end{abstract}

\section{INTRODUCTION}

It has been established based on experimental data that supersonic jet noise consists of multiple components [1, 2], including turbulent mixing noise, Mach wave radiation, broadband shock-associated noise and, under certain conditions, the so-called screech and crackle noise. Some of these components radiate in the same direction, and are

*PhD, now at SNECMA, email: nicolas.decacqueray@gmail.com †CNRS Research Scientist, email: christophe.bogey@ec-lyon.fr 
affected, because of their high intensity, by non-linear propagation effects. This can cause difficulties to quantify their relative contributions to the acoustic far field, and to identify their generation mechanisms.

Some of these issues may be addressed by using simulations, which have made, thanks to the increase in computational resources as well as to the development of lowdispersion and low-dissipation numerical schemes, spectacular progress over the last two decades. It is thus now possible to solve the compressible unsteady Navier-Stokes equations to compute simultaneously the flow and acoustic fields of subsonic jets $[3,4$, $5,6,7,8,9]$ and supersonic jets $[10,11,12,13,14,15,16]$. Such simulations have enabled, among other things, to predict noise without empirical model, to perform investigations on jet sound sources, and to study the influence of nozzle-exit conditions.

In a previous work by the authors [13], an overexpanded supersonic round jet was computed using large-eddy simulation (LES). At the exit section of a straight pipe nozzle of radius $r_{e}$, the jet is laminar and has a Mach number of $M_{e}=3.3$, a temperature of $T_{e}=360 \mathrm{~K}$, a static pressure of $p_{e}=0.5 \times 10^{5} \mathrm{~Pa}$, and a diameter-based Reynolds number of $\operatorname{Re} \simeq 10^{5}$. The properties of the turbulent flow field as well as the acoustic near field of the jet were characterized using, in particular, azimuthal decompositions. The properties of the acoustic field propagated to a distance of $80 r_{e}$ from the nozzle exit by solving the full Euler equations were described in the same way. The contributions of Mach waves, turbulent mixing noise, broadband shockassociated noise, and screech noise to the far field were identified by showing connections between the turbulent flow field and the acoustic near and far fields.

In the present paper, the data obtained by LES for this jet at $M_{e}=3.3$ are re-examined. The first objective is to investigate the non-linear propagation effects on the sound waves emitted by the jet. Non-linear propagation effects are indeed important for supersonic jets, for turbojet [17] and tactical [18] aircrafts as well as for heated supersonic jets considered in laboratory experiments [19]. They result in an energy transfer from the spectral peak to the higher frequencies as the propagation distance increases. Their impact on Mach wave radiation, broadband shock-associated noise and crackle noise has been explored in recent studies [16, 20, 21, 22, 23, 24, 25] by propagating spectra to the same distance linearly or by solving the non-linear Burgers equation, and by estimating indicators of nonlinearity such as the skewness values of pressure signals. Here, two far-field wave extrapolations are performed from the LES near-field data by solving the linearized Euler equations or the full Euler equations up to a distance of $240 r_{e}$ from the nozzle exit. The non-linear propagation effects are quantified by comparing the results of these two calculations for different propagation distances in both time and frequency domains.

The second objective of the paper is to discuss noise generation mechanisms by calculating cross-correlations between the far-field pressure fluctuations and turbulent quantities, such as axial velocity fluctuations, vorticity norm and density fluctuations, recorded on the jet axis and along the shear layer in the LES. This method, which has been proposed in the seventies, has recently been applied to subsonic jets [26, 27, 28, $29,30]$ as well as to supersonic jets [31, 32, 33, 34]. It permits us to identify statistical 
correlations between the flow field and the acoustic field, which can be attributed to noise generation when they are found for time delays consistent with a propagation at the speed of sound between the points considered.

The paper is organized as follows. The main parameters as well as some results of the jet LES are given in section 2. The non-linear propagation effects on the sound waves emitted by the jet are studied in section 3. Normalized cross-correlations calculated between far-field pressure fluctuations and turbulent quantities in the jet are presented in section 4. Finally, concluding remarks are provided in section 5.

\section{LARGE-EDDY SIMULATION OF THE MACH 3.3 JET}

In this section, the main parameters of the jet simulation whose data are used in the present study are provided. The key features of the jet flow field are also shown. More results are available in de Cacqueray et al. [13]

\subsection{Parameters}

An overexpanded jet has been computed in a previous work [13]. At the exit section of a nozzle of radius $r_{e}=1.6 \mathrm{~mm}$, at $z=0$, it is characterized by a Mach number of $M_{e}=3.30$, a temperature of $T_{e}=360 \mathrm{~K}$ and a static pressure of $p_{e}=0.5 \times 10^{5} \mathrm{~Pa}$, all three of which are very similar to the exit quantities of the jet considered in the experiment of Varnier \& Gély [35].

The jet radius is however about 20 times smaller in the simulation due to LES constraints. The stagnation pressure and temperature are $28.6 \times 10^{5} \mathrm{~Pa}$ and $1144 \mathrm{~K}$ yielding, given the specific heat ratio of $\gamma=1.4$ imposed in the simulation, an exit velocity of $u_{e}=1255 \mathrm{~m} \cdot \mathrm{s}^{-1}$. The equivalent fully-expanded exit conditions obtained for the same stagnation conditions and a static pressure of $p_{j}=10^{5} \mathrm{~Pa}$ are a Mach number of 2.83, a temperature of $439 \mathrm{~K}$ and a radius of $r_{j}=0.81 r_{e}$. The acoustic Mach number $M_{a}$, defined as the ratio between the fully-expanded velocity $u_{j}=1190 \mathrm{~m} \cdot \mathrm{s}^{-1}$ and the sound speed in the ambient medium $c_{\infty}=343 \mathrm{~m} . \mathrm{s}^{-1}$, is equal to 3.47. The Reynolds number calculated from the nozzle-exit quantities is $R_{e}=2 r_{e} u_{e} \rho_{e} / \mu_{e}=0.94 \times 10^{5}$, where $\rho_{\mathrm{e}}$ and $\mu_{e}$ are the density and molecular viscosity at the jet exit. The nozzle is a straight pipe of length $0.5 r_{e}$, whose lip is $0.05 r_{e}$ thick. Inside the pipe, a Blasius profile for a laminar boundary layer of thickness $\delta=0.05 r_{e}$ is imposed for the mean velocity, and a Crocco-Busemann relation is used to determine the mean density profile. Random pressure disturbances of low amplitude are also added in the nozzle, which results in nozzle-exit maximum velocity fluctuations of about $1 \%$ of the jet velocity.

The simulation has been performed by solving the unsteady compressible NavierStokes equations in cylindrical coordinates, using low-dispersion and low-dissipation explicit finite-difference schemes [36, 37, 38], namely 11-point 4th-order finite differences and 6th-order filter for space discretization, and a 2nd-order 6-stage RungeKutta algorithm for time integration. Near the jet centerline, the axis singularity is treated using the method proposed by Mohseni \& Colonius [39], and the effective azimuthal resolution is reduced [40] in order to increase the time step. The LES approach is based on the explicit application of a relaxation filtering to the flow variables [41] to take into 
account the dissipative effects of the subgrid scales. Non-reflective acoustic boundary conditions are implemented, in combination with a sponge sponge at the outflow boundary [42]. In addition, a shock-capturing method using dilatation for the shock detection and a 2nd-order filter is applied in order to remove Gibbs oscillations near shocks [38]. The mesh grid contains $n_{r} \times n_{\theta} \times n_{z}=256 \times 128 \times 840=28 \times 10^{6}$ points, and extends axially down to $z=52 r_{e}$. After the initial transient period of the computation, the LES data obtained at $r=0$ as well as on the cylindrical surfaces located at $r=r_{j}$ and $r=9.5 r_{e}$ have been recorded during a time period of $1323 r_{e} / u_{e}$.

\subsection{Snapshots and jet flow field}

Snapshots of the norm of the density gradient and of azimuthal vorticity in the jet, and of the fluctuating pressure outside, are shown in figure 1. In the jet, a shock-cell structure is clearly found, and the turbulent development of the flow can be observed. Outside, strong acoustic waves traveling in the downstream direction as well as upstream-propagating waves corresponding to broadband shock-associated noise and screech noise [13] are visible.

The centerline variations of the mean static pressure normalized by the ambient pressure and of the inverse of the mean velocity scaled by the jet exit velocity are represented in figures 2(a) and 2(b). In the first figure, six shock cells resulting from the adjustment of the jet exit static pressure to the ambient pressure are observed. The amplitude of the pressure oscillations decreases with the axial position, as expected. In

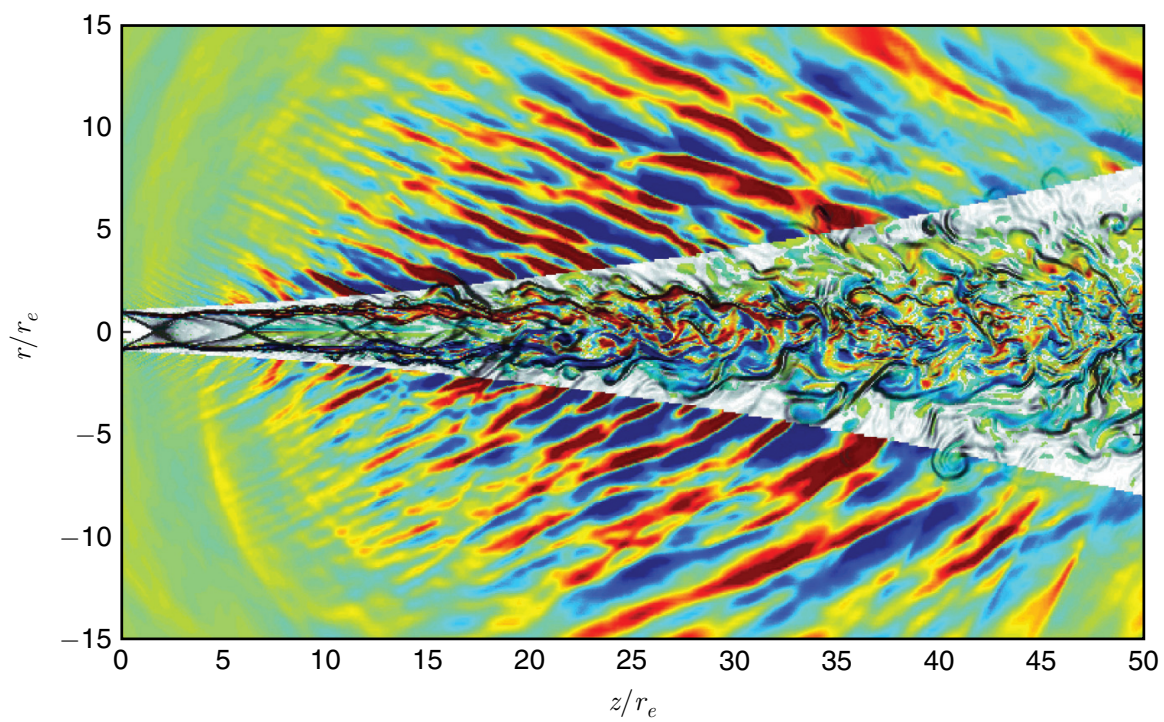

Figure 1: Snapshots in the $(z, r)$ plane of the norm of the density gradient and of azimuthal vorticity in the jet, and of fluctuating pressure $p^{\prime}$ outside. The color scale ranges from $-5000 \mathrm{~Pa}$ to $5000 \mathrm{~Pa}$ for $p^{\prime}$. 
(a)

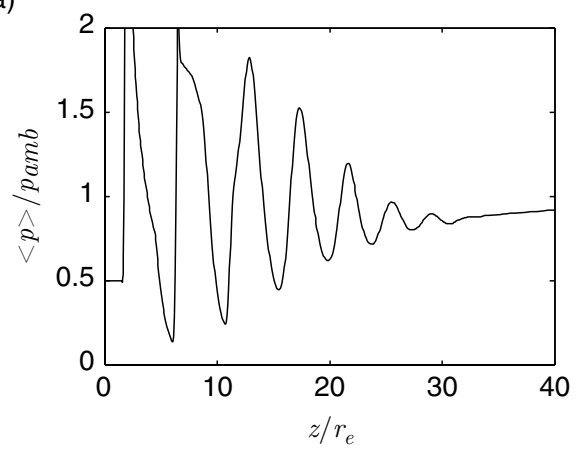

(b)

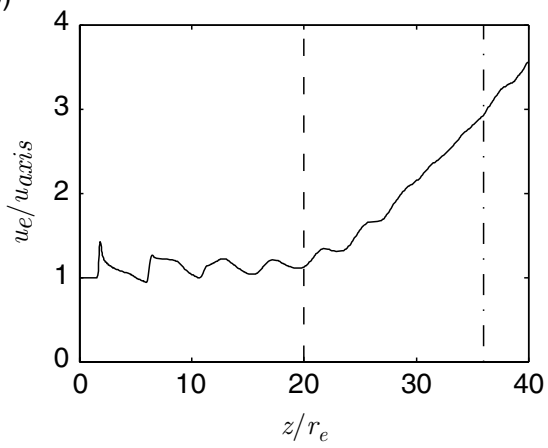

Figure 2: $\quad$ Centerline variations of (a) mean static pressure $\langle p\rangle$, and (b) the inverse of mean longitudinal velocity $u_{\text {axis }}$; positions of --- the end of the potential core $z_{c}=20 r_{e}$, and --- the end of the sonic core $z_{s}=36 r_{e}$.

the second figure, the mean axial velocity is seen to be modulated by the shock-cell structure. The end of the potential core and that of the sonic core, estimated using the criteria $u_{\text {axis }}=0.9 u_{e}$ and $u_{\text {axis }}=c$, respectively, where $c$ is the local speed of sound, are found at $z_{c}=20 r_{e}$ and $z_{s}=36 r_{e}$ from the nozzle exit. The core lengths in the present jet are shorter than those obtained in the experiment of Varnier \& Gély [35] for a jet with similar exit quantities $M_{e}, p_{e}$ and $T_{e}$, and a higher Reynolds number of $R_{e}=1.75 \times 10^{6}$, where $z_{c}=24 r_{e}$ and $z_{s}=50 r_{e}$ have been measured. This may be due to the laminar nozzle-exit conditions [7,9] in the simulation.

The variations of the rms values of axial and radial velocity fluctuations $u_{z}^{\prime}$ and $u_{r}^{\prime}$ and of density fluctuations $\rho^{\prime}$ at $r=0$ and $r=r_{j}$ are presented in figures 3(a) and 3(b). On the jet centerline, in figure 3(a), the rms velocity fluctuations are weak between $z=0$ and $z=10 r_{e}$, and then increase with the axial distance to reach maximum values around $z=25 r_{e}$ downstream of the end of the potential core. The rms values of $\rho^{\prime}$ also

(a)

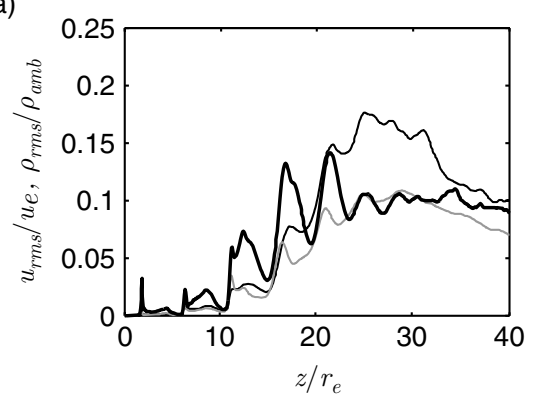

(b)

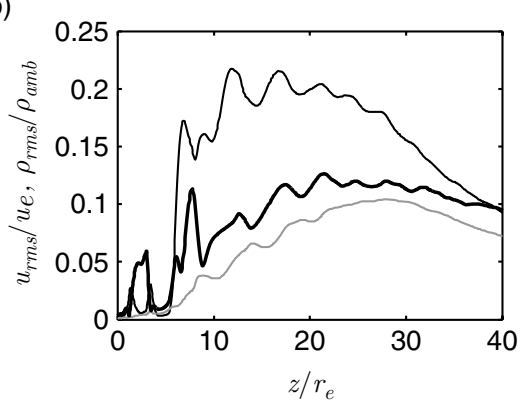

Figure 3: Axial variations of the $r m s$ values of velocity and density fluctuations at (a) $r=0$ and (b) $r=r_{j}:-u_{z}^{\prime},-u_{r}^{\prime},-\rho^{\prime}$. 
(a)

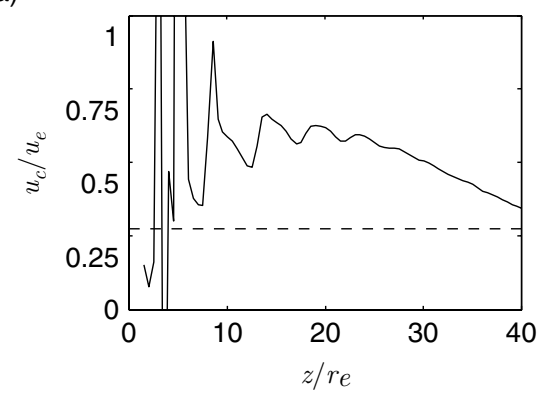

(b)

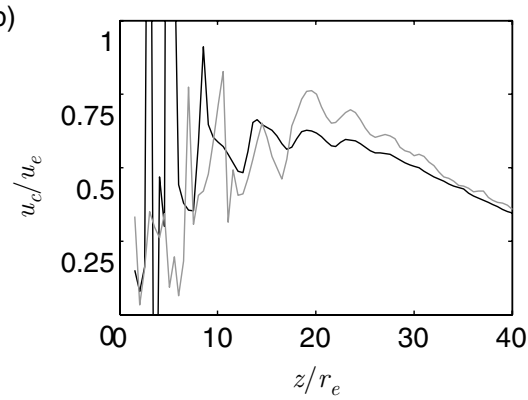

Figure 4: Axial variations of the convection velocity $u_{c}$ obtained from crosscorrelations of axial velocity fluctuations: (a) $u_{c} / u_{e}$ at $r=r_{j}$ and $--c_{a m b} / u_{e},(\mathrm{~b})-u_{c} / u_{e}$ at $r=r_{j}$ and $-u_{c} / u_{e}$ at $r=0$.

increase in the jet potential core, but they appear more disturbed by the shock-cell structure, in particular between $z=10 r_{e}$ and $z=27 r_{e}$. At $r=r_{j}$ along the shear layer, in figure 3(b), the peak rms value of velocity $u_{z}^{\prime}$ is found around $z=15 r_{e}$ upstream of the end of the jet potential core, whereas the peak rms values of $u_{r}^{\prime}$ and ${ }^{\prime} \rho$ are located farther downstream around $z=25 r_{e}$.

The convection velocity $u_{c}$ of turbulent structures along the line $r=r_{j}$ is presented in figure 4(a). It is calculated from the cross-correlations of axial velocity fluctuations $u_{z}^{\prime}$, and is normalized by the jet exit velocity $u_{e}$. Just downstream of the nozzle exit, the convection velocity is strongly modulated by the shock-cell structure, and may not be very accurate. It can however be noted that the convection velocity is close to $u_{c}=0.6 u_{e}$ between $z \simeq 10 r_{e}$ and $z \simeq 25 r_{e}$, and then decreases farther downstream. By comparing with the ambient sound speed $c_{a m b}$ represented by a dashed line in figure 4(a), it also appears that the convection velocity at $r=r_{j}$ is supersonic for $z>$ $5 r_{e}$. Mach waves are consequently expected to be generated in the present jet.

The convection velocity at $r=r_{j}$ is replotted along with that obtained on the jet centerline in figure 4(b). The latter is higher than the former for $z \geq 17 r_{e}$. In that flow region, the convection velocity at $r=0$ increases to reach a peak value equal to $75 \%$ of the jet exit velocity at $z=19.5 r_{e}$, that is near the end of the potential core, and then decreases farther downstream.

\section{NON-LINEAR NOISE PROPAGATION EFFECTS}

\subsection{Far-field wave extrapolations}

In order to investigate the non-linear propagation effects on the sound waves emitted by the jet, two far-field wave extrapolations are carried out using the LES data recorded on the cylindrical surface at $r=9.5 r_{e}$ extending down to $z=52 r_{e}$. The extrapolations are performed by solving the isentropic linearized Euler equations in the first case and the full non-linear Euler equations in the second case. The effects of atmospheric absorption are not considered in order to focus on non-linear propagation effects. 
Moreover, if they had been taken into account, they would have been, because of the small jet radius of $r_{e}=1.6 \mathrm{~mm}$ chosen to ensure LES accuracy, very strong and, in particular, much stronger than those encountered experimentally. The numerical methods, except for the filter used in the shock-capturing procedure, are identical to those employed for the LES. For the shock-capturing filtering, a standard 4th-order filter is implemented instead of a 2nd-order filter, which is sufficient to handle the weak shock waves which are formed during the sound propagation. The simulations are performed on a cylindrical grid containing $n_{r} \times n_{\theta} \times n_{z}=2250 \times 64 \times 1950=280$ $\times 10^{6}$ points, onto which the LES data are imposed at the bottom boundary at $r=9.5 r_{e}$. The radial and axial mesh spacings are uniform with $\Delta r=\Delta z=0.1 r_{e}$, yielding a Strouhal number of $S t_{e}=f \times\left(2 r_{e}\right) / u_{e}=1.5$ for an acoustic wave discretized by 4 points per wavelength, where $f$ is the frequency. In order to highlight the cumulative nonlinear distortion of the acoustic signals during propagation, pressure is recorded at different distances from the nozzle exit for the angle of $\phi=60^{\circ}$ with respect to the jet direction during a time period around $1220 r_{e} / u_{e}$ in each case. Spectra are averaged in the azimuthal direction.

Snapshots of pressure obtained at the same time in the two computations based on the linearized and the full Euler equations are provided in figures 5(a) and 5(b). The radiation angle of $\phi=60^{\circ}$ relative to the jet direction is represented by a dashed line, and the distances of $d=60 r_{e}, 120 r_{e}, 180 r_{e}$ and $240 r_{e}$ from the nozzle exit are

(a)

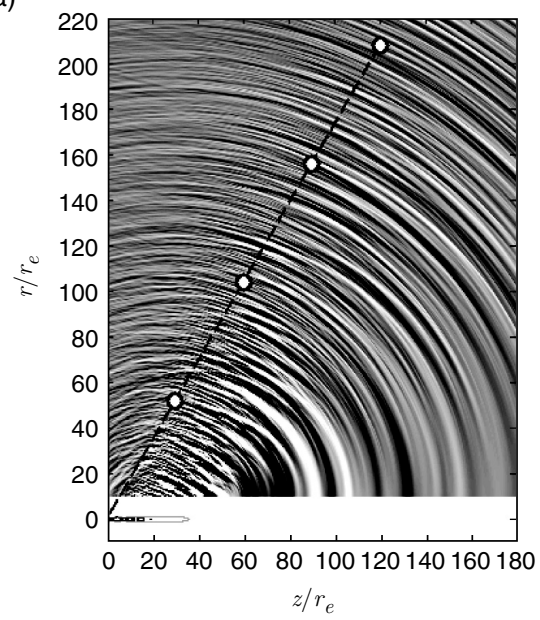

(b)

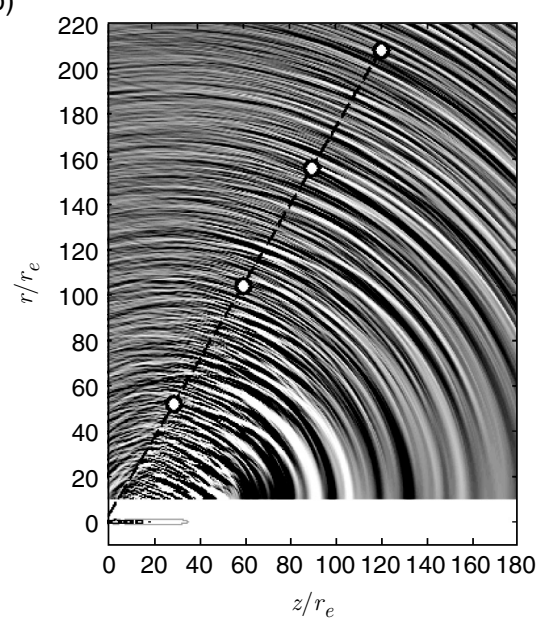

Figure 5: Snapshots of pressure fluctuations obtained from the far-field wave extrapolations based on (a) the linearized and (b) the full Euler equations, using a grey scale ranging from $-600 \mathrm{~Pa}$ to $600 \mathrm{~Pa}$. Representation of the isocontours $-<u_{z}>/ u_{e}=0.9$ and $-<u_{z}>/ c_{a m b}=1$ in the jet, --- the angle $\phi=60^{\circ}$ and o the distances $d=60 r_{e}, 120 r_{e}, 180 r_{e}$ and $240 r_{e}$ from the nozzle exit. 


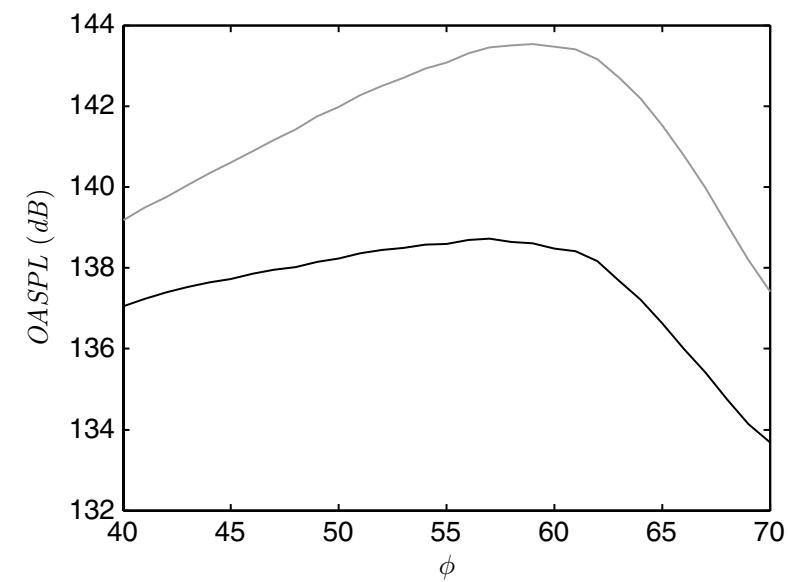

Figure 6: Overall sound pressure levels obtained at $d=240 r_{e}$ from the far-field wave extrapolations based on the linearized and the full Euler equations.

indicated by white circles on that line. In both figures, strong pressure waves are observed to propagate in the downstream direction. Their levels seem however slightly higher in the linear case in figure 5(a) than in the non-linear case in figure 5(b). In order to give a glimpse of the origin of the acoustic waves in the jet, the contour lines corresponding to mean axial velocities of $\left\langle u_{z}\right\rangle=0.9 u_{e}$ and $\left\langle u_{z}\right\rangle=$ $c$, where $c$ is the local sound speed, are depicted below the pressure fields. Most of the sound waves appear to be generated upstream of the end of the sonic core located at $z_{s}=36 r_{e}$.

The overall sound pressure levels estimated at the distance $d=240 r_{e}$ from the nozzle exit are shown in figure 6 as a function of the angle $\phi$ defined in degrees with respect to the jet direction. They are calculated by integrating the pressure spectra from Strouhal number $S t_{e}=0.023$ up to $S t_{e}=1$. For all the radiation angles $40^{\circ} \leq \phi \leq 70^{\circ}$ considered, the noise levels obtained by solving the full Euler equations are lower than those obtained using the linearized Euler equations. In particular, the peak level observed around $\phi=60$ is reduced by about $5 \mathrm{~dB}$. Non-linear propagation effects thus appear to be quite significant on the far-field noise of the present jet.

\subsection{Non-linear effects along the line $\phi=60^{\circ}$}

Non-linear effects in the propagation of high-speed jet noise obviously depend on the radiation angle, as illustrated in Gee et al. [18] for example. In the present paper, they are investigated by comparing the results obtained using linear and non-linear wave extrapolations for the angle $\phi=60^{\circ}$, which is close to the peak directivity angle in figure 6 . The signals of pressure fluctuations recorded at $d=60 r_{e}, 120 r_{e}, 180 r_{e}$, and $240 r_{e}$ from the nozzle exit are represented in figures 7(a-d) as a function of time $t-\tau$ normalized by $u_{e} /\left(2 r_{e}\right)$, where $\tau=d / c_{a m b}$. In figure $7(\mathrm{a})$, the pressure signals 
(a)

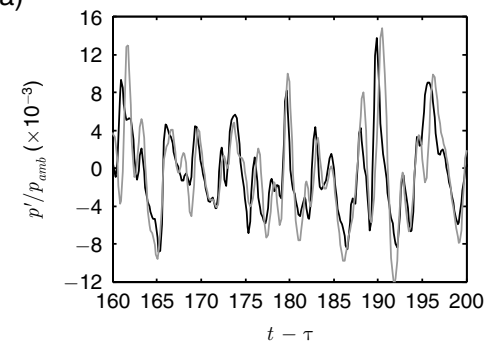

(c)

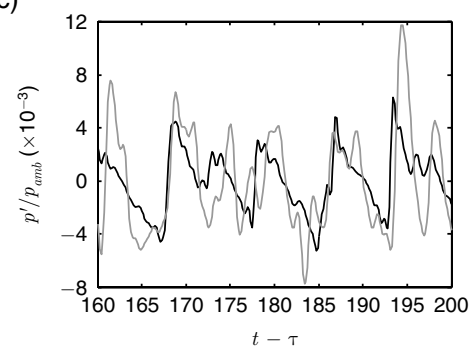

(b)

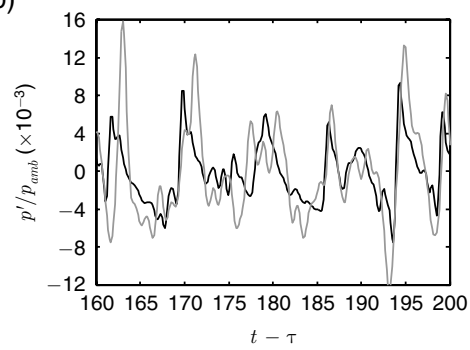

(d)

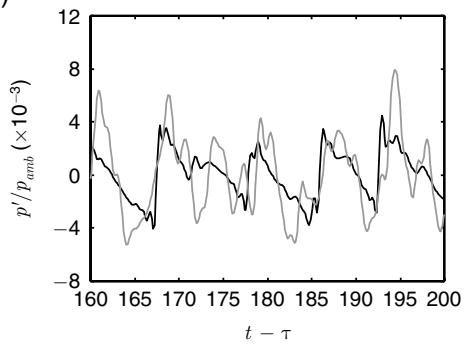

Figure 7: Signals of pressure fluctuations obtained for $\phi=60^{\circ}$ at (a) $d=60 r_{e}$, (b) $d=120 r_{e}$, (c) $d=180 r_{e}$ and (d) $d=240 r_{e}$ from the nozzle exit from the far-field extrapolations based on — the linearized and the full Euler equations. Time $t$ and time delay $\tau=d / c_{a m b}$ are normalized by $u_{e} /\left(2 r_{e}\right)$.

determined at $d=60 r_{e}$ from the two acoustic computations are similar. Differences due to non-linear effects can however be observed, as is the case at time $t-\tau \simeq 190$ for instance. As the propagation distance from the nozzle exit increases, the changes between the two signals are stronger. The pressure signal calculated using the full Euler equations gradually has a lower amplitude than that calculated using the linear Euler equations. Weak shock waves also appear in the non-linear case, leading to a series of $N$-shaped waves in the signal at $d=240 r_{e}$ in figure 7(d). The acoustic waves are therefore strongly distorted during propagation.

The non-linear propagation effects are now examined in the frequency domain. The pressure spectra obtained at the four positions considered in figures $7(\mathrm{a}-\mathrm{d})$, for $\phi=60^{\circ}$ and $d=60 r_{e}, d=120 r_{e}, d=180 r_{e}$ and $d=240 r_{e}$ from the nozzle exit, are plotted in figures $8(\mathrm{a}-\mathrm{d})$ as a function of the Strouhal number $S t_{e}$. They are represented up to $S t_{e}=1$, because of the cut-off Strouhal numbers of $S t_{e}=1.37$ and $S t_{e}=1.5$ imposed by the grids in the LES and in the far-field extrapolations, respectively. In figure 8(a), the spectra calculated at $d=60 r_{e}$ using linear and non-linear wave extrapolations are similar for $S t_{e} \leq 0.1$, but they differ appreciably for higher frequencies. Compared to the linear case, the noise levels in the non-linear case are weaker for $0.1<S t_{e}<0.9$ and stronger for $S t_{e}>0.9$. Farther away from the nozzle exit, 
(a)

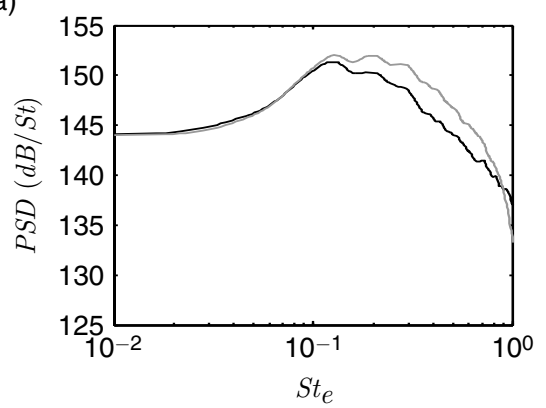

(c)

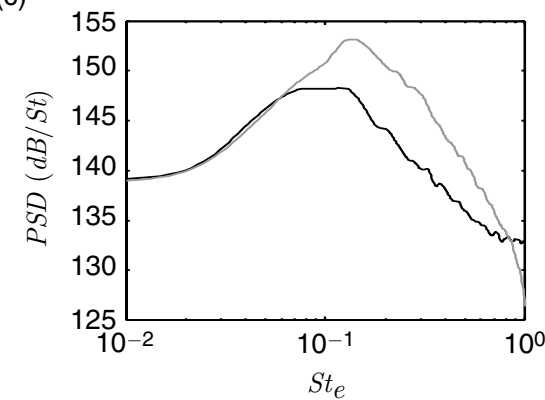

(b)

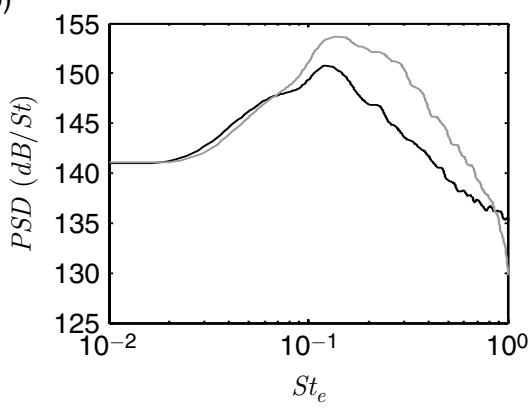

(d)

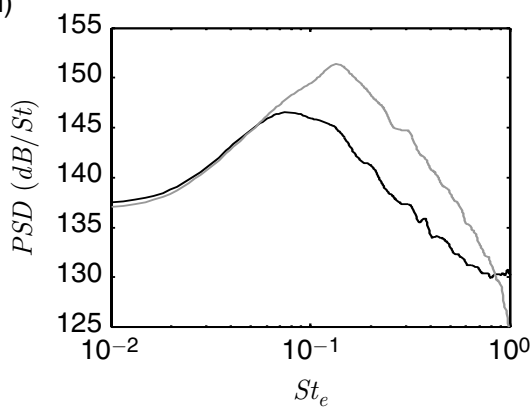

Figure 8: Spectra of pressure fluctuations obtained for $\phi=60^{\circ}$ at (a) $d=60 r_{e}$, (b) $d=120 r_{e}$, (c) $d=180 r_{e}$ and (d) $d=240 r_{e}$ from the nozzle exit from the far-field extrapolations based on — the linearized and — the full Euler equations, as a function of $S t_{e}=f \times\left(2 r_{e}\right) / u_{e}$

the differences between the spectra become more important as non-linear propagation effects accumulate. Due to these effects, the peak level is reduced, and is reached at a frequency moving to lower Strouhal numbers. Moreover, the contributions of highfrequency components increase, leading to higher noise levels for $S t_{e}>0.85$ at $240 r_{e}$ from the nozzle in figure $8(\mathrm{~d})$. This energy transfer from mid frequencies to high frequencies is typical of non-linear propagation effects, see for instance in the works by Gee et al. [18], Petitjean et al. [20], Viswanathan [19] and Baars et al. [23], among others.

In order to quantify the gains and losses on noise levels due to non-linear effects, the differences between the pressure spectra obtained using non-linear and linear wave extrapolations for $\phi=60^{\circ}$ at $d=60 r_{e}, 120 r_{e}, 180 r_{e}$ and $240 r_{e}$ from the nozzle are displayed in figure 9. In all cases, they do not exceed $1 \mathrm{~dB}$ for $S t_{e} \leq 0.06$, but they are significant for higher Strouhal numbers. For $0.06<S t_{e}<0.85$, the differences between the spectra are negative, and grow with the distance from the nozzle exit, indicating a gradual loss of energy during non-linear propagation. The 


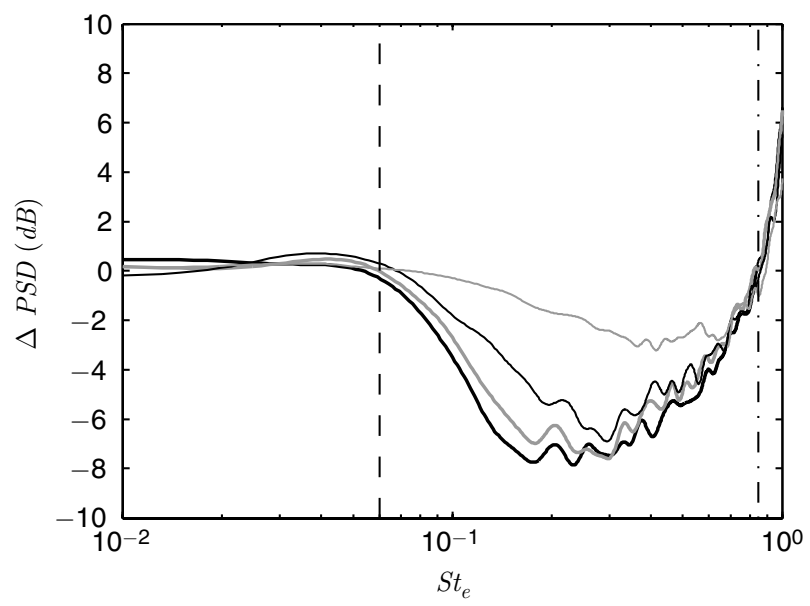

Figure 9: Differences between the spectra of pressure fluctuations obtained using the full and the linearized Euler equations for $\phi=60^{\circ}$ at $-d=60 r_{e}$, $-d=120 r_{e},-d=180 r_{e}$ and $-d=240 r_{e}$ from the nozzle exit; - - $S t_{e}=0.06,-\cdot-S t_{e}=0.85$, with $S t_{e}=f \times\left(2 r_{e}\right) / u_{e}$.

maximum loss is also found for lower frequencies with increasing $d$. At $d=240 r_{e}$, for instance, it reaches a value of $-7.8 \mathrm{~dB}$ at $S t_{e} \simeq 0.23$. For Strouhal numbers $S t_{e}>$ 0.85 , the differences between the spectra are positive, and a gain of about $6 \mathrm{~dB}$ is achieved at $S t_{e}=1$. The noise components in this frequency range are therefore strengthened as energy is transferred from mid to high frequencies due to nonlinearity. Finally, it can be noted that the curves obtained at $d=180 r_{e}$ and $d=240 r_{e}$ are close. This suggests that non-linear propagation effects are weak at very large distances from the nozzle exit.

\section{CORRELATIONS BETWEEN FLOW AND FAR-FIELD NOISE 4.1. Definitions}

Noise generation is investigated by computing correlations between turbulent quantities in the jet flow and far-field pressure fluctuations. The turbulent quantities considered are the axial velocity fluctuations $u_{z}^{\prime}$, the normal stress in the radial direction $u_{r}^{\prime} u_{r}^{\prime}$, the norm of the vorticity $|\omega|$ and the density fluctuations $\rho^{\prime}$ obtained at $r=0$ and at $r=r_{j}$ in the LES. The pressure fluctuations $p^{\prime}$ are those calculated from the far-field wave extrapolation based on the full Euler equations for the radiation angle of $\phi=60^{\circ}$ at $d=60 r_{e}, 120 r_{e}, 180 r_{e}$ and $240 r_{e}$ from the nozzle exit, at the locations indicated by the white circles in figure 5(b). The normalized correlations between the turbulent quantities at position $\mathrm{x}_{1}$ in the jet and the pressure fluctuations at distance $d$ are evaluated in the following way: 


$$
\begin{aligned}
C u_{z} p\left(\mathrm{x}_{1}, d, \tau\right) & =\frac{<u_{z}^{\prime}\left(\mathrm{x}_{1}, t\right) p^{\prime}(d, t+r)>}{<u_{z}^{\prime 2}\left(\mathrm{x}_{1}, t\right)>^{1 / 2}<p^{\prime 2}(d, t)>^{1 / 2}} \\
C u_{r}^{2} p\left(\mathrm{x}_{1}, d, \tau\right) & =\frac{<\left(u_{r}^{\prime 2}\left(\mathrm{x}_{1}, t\right)-<u_{r}^{\prime 2}\left(\mathrm{x}_{1}, t\right)>\right) p^{\prime}(d, t+\tau)>}{<\left(u_{r}^{\prime 2}\left(\mathrm{x}_{1}, t\right)-<u_{r}^{\prime 2}\left(\mathrm{x}_{1}, t\right)>\right)^{2}>^{1 / 2}<p^{\prime 2}(d, t)>^{1 / 2}} \\
C|\omega| p\left(\mathrm{x}_{1}, d, \tau\right) & =\frac{<|\omega|\left(\mathrm{x}_{1}, t\right) p^{\prime}(d, t+\tau)>}{\left.<|\omega|^{2}\left(\mathrm{x}_{1}, t\right)>^{1 / 2}<p^{\prime 2}(d, t)\right\rangle^{1 / 2}} \\
C \rho p\left(\mathrm{x}_{1}, d, \tau\right) & =\frac{<\rho^{\prime}\left(\mathrm{x}_{1}, t\right) p^{\prime}(d, t+\tau)>}{\left.<\rho^{\prime 2}\left(\mathrm{x}_{1}, t\right)>^{1 / 2}<p^{\prime 2}(d, t)\right\rangle^{1 / 2}}
\end{aligned}
$$

where $<$. $>$ denotes time averaging, and $\tau$ is the time delay between the flow and acoustic signals. The position $\mathrm{x}_{1}$ is equal to $(r=0, z)$ along the jet centerline, and to $\left(r=r_{j}, z\right)$ along the shear layer. The correlations are averaged in the azimuthal direction to enhance convergence.

\subsection{Correlations with centerline flow quantities}

The correlations calculated between the signals of $u_{z}^{\prime}, u_{r}^{\prime} u_{r}^{\prime}$, $|\omega|$ and $\rho^{\prime}$ obtained for $0 \leq z / r_{e} \leq 40$ on the jet centerline, and the pressure fluctuations at $\phi=60^{\circ}$ and $d=60 r_{e}, 120 r_{e}, 180 r_{e}$ and $240 r_{e}$, are first examined. They are represented in figure 10 as functions of $z / r_{e}$ and of the time delay $\tau$ normalized by $u_{e} /\left(2 r_{e}\right)$. The positions of $z_{c}=20 r_{e}$ and $z_{s}=36 r_{e}$, where the potential and the sonic cores end, and the time delay corresponding to a linear propagation at the ambient speed of sound along straight lines between the centerline and the far-field points, are also indicated. For all flow quantities, the correlations are weak for the pressure fluctuations at $d=60 r_{e}$ from the nozzle in figure 10(i). However, they strengthen with increasing propagation distance, and significant levels of correlations are found for large values of $d$. They are located near the acoustic time delay, which suggests a link with noise generation. Moreover, the correlation maps obtained for points at $d=180 r_{e}$ and $240 r_{e}$ in figures 10(iii) and 10(iiii) look very alike, supporting geometrical convergence of the acoustic far field.

The correlations obtained between the centerline quantities and the pressure fluctuations at $d=240 r_{e}$ are described in more detail. Strong correlations are observed downstream of the potential core in figure 10(a)(iiii) for the axial velocity fluctuations, around the end of the potential core at $z_{c}=20 r_{e}$ in figures 10(b)(iiii) and 10(c)(iiii) for the radial radial stress and the vorticity norm, and between $z=12$ and $40 r_{e}$ in figure 10 (d)(iiii) for the density fluctuations. In the latter figure, the sign of the correlations changes with the axial position, which is not the case in the three former figures. It can also be noted that for $u_{z}^{\prime}$ and $|\omega|$, the correlation maps are similar to those obtained for subsonic round jets and hot coaxial jets in Bogey \& Bailly [27] and Bogey et al. [28]. 
(a)
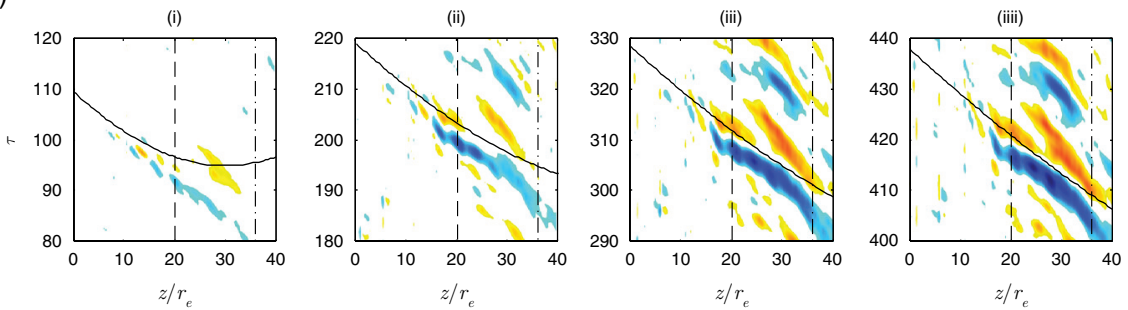

(b)
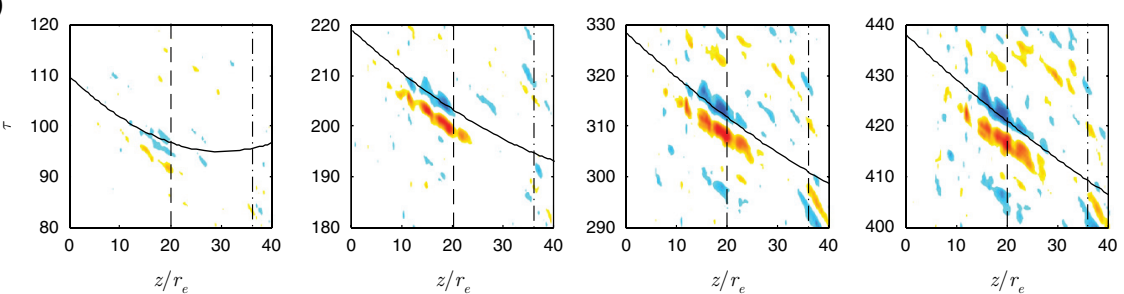

(c)
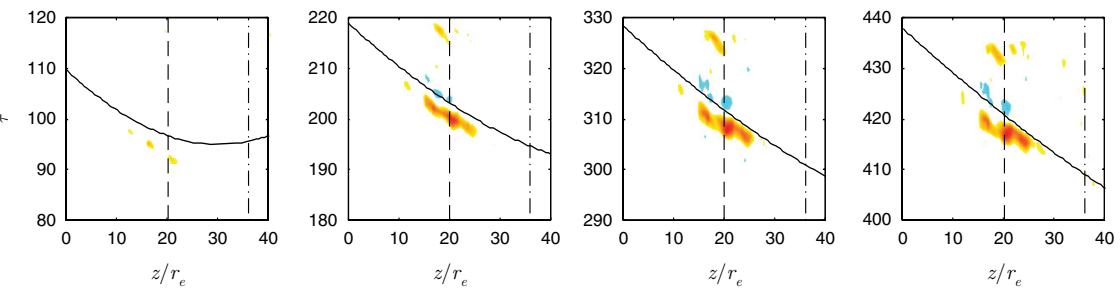

(d)
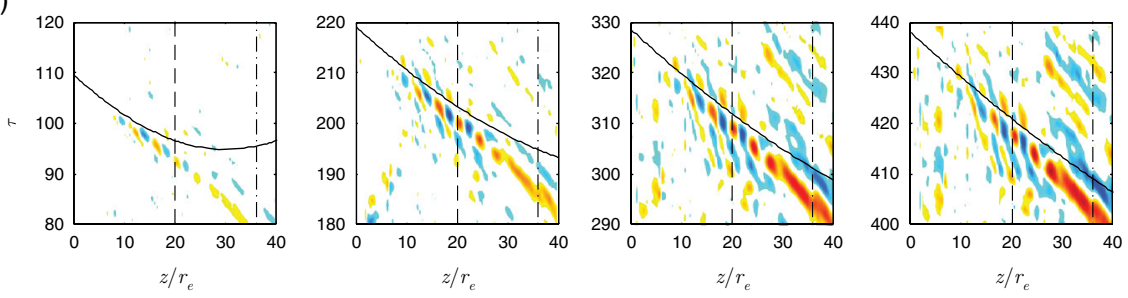

Figure 10: Normalized correlations between pressure fluctuations $p^{\prime}$ obtained for $\phi=60^{\circ}$ at (i) $d=60 r_{e}$, (ii) $d=120 r_{e}$, (iii) $d=180 r_{e}$ and (iiii) $d=240 r_{e}$ from the nozzle exit using non-linear wave extrapolation, and centerline flow quantities (a) $u_{z}^{\prime}$, (b) $u_{r}^{\prime} u_{r}^{\prime}$, (c) $|\omega|$ and (d) $\rho^{\prime}$ (X-axis: — position on the jet centerline, Y-axis: time delay normalized by $\left.u_{e} /\left(2 r_{e}\right)\right)$; time delay for a rectilinear propagation at the speed of sound, --- end of the potential core, - - - end of the sonic core. The color scales range from -0.25 to 0.25 for $(\mathrm{a}, \mathrm{d})$ and from -0.15 to 0.15 for $(\mathrm{b}, \mathrm{c})$. 
More quantitative results are provided for the correlations $C u_{z} p$ and $C|\omega| p$ computed from the centerline velocity fluctuations and vorticity norm. The axial variations of the peak absolute values of these correlations are plotted in figures 11(ab) for the pressure signals at $d=60 r_{e}, 120 r_{e}, 180 r_{e}$ and $240 r_{e}$. In all cases, they show oscillations, which can be associated with the shock-cell structure in the jet plume. In figure 11(a), as already remarked from the correlation maps, the levels obtained for $\left|C u_{z} p\right|$ increase with the propagation distance, thus reaching maximum values of 0.11 , $0.19,0.23$ and 0.26 for the four propagation distances considered. The peak location moreover shifts in the downstream direction, from $z \simeq 14 r_{e}$ for $d=60 r_{e}$ down to $z$ $\simeq 25 r_{e}$ for $d=240 r_{e}$. The latter position roughly agrees with that of the maximum $r m s$ value of centerline axial velocity fluctuations in figure 3(a). In figure 11(b), the levels obtained for $|\mathrm{C}| \omega|p|$ also rise with the propagation distance. In that case, however, the strongest correlations are found at positions which do not vary much with $d$, and correspond approximatively to the position of the end of the potential core for $120 r_{e} \leq d \leq 240 r_{e}$. At $d=240 r_{e}$, for instance, the maximum value is reached at $z=20.5 r_{e}$, and is equal to 0.11 .

The normalized correlations calculated between $u_{z}^{\prime}$ and $|\omega|$ at $r=0$ and $z=20.5 r_{e}$, that is just downstream of the potential core, and the pressure fluctuations at $\phi=60^{\circ}$ and $d=240 r_{e}$ are represented in figures 12(a-b) as a function of the time delay between the flow and acoustic signals. In both cases, a peak correlation is observed for a time delay slightly inferior to the time estimated for a propagation at the speed of sound. The peak value is negative for $\mathrm{C} u_{z} p$ in figure 12(a) and positive for $C|\omega| p$ in figure 12(b).

The properties of the flow quantities $u_{z}^{\prime}$ and $|\omega|$ on the jet axis are discussed in order to shed light on the possible cause of the correlations with the far-field pressure reported above. The axial variations of the skewness factor of the axial velocity fluctuations are first plotted in figure 13(a). Large negative values are found for $15 r_{e} \leq z \leq 25 r_{e}$, with

(a)

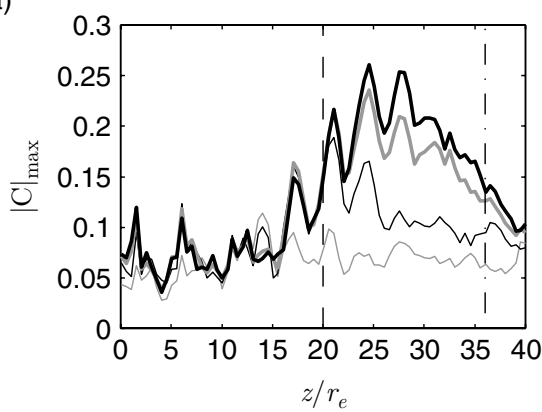

(b)

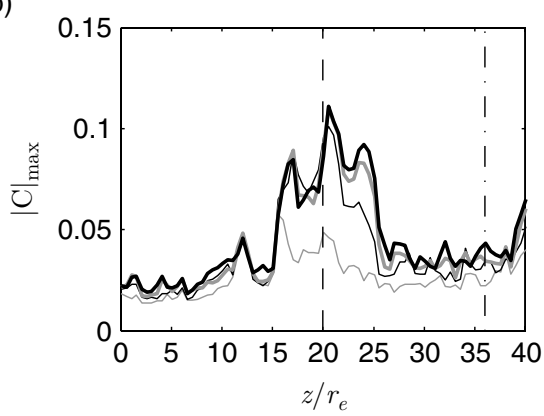

Figure 11: Axial variations of the peak absolute value of normalized correlations between centerline flow quantities (a) $u_{z}^{\prime}$ and (b) $|\omega|$, and pressure fluctuations at $\phi=60^{\circ}$ and $-d=60 r_{e},-d=120 r_{e}$, $d$ $=180 r_{e}$ and $-d=240 r_{e}$ from the nozzle exit; --- end of the potential core, -.-.- end of the sonic core. 
(a)

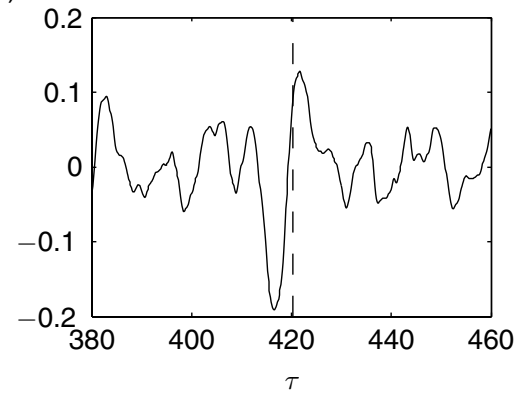

(b)

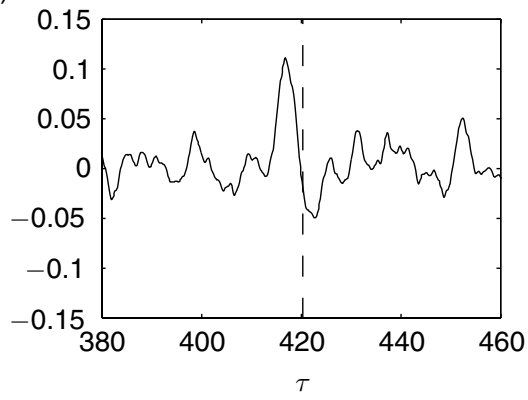

Figure 12: Normalized correlations between (a) $u_{z}^{\prime}$ and (b) $|\omega|$ at $z=20.5 r_{e}$ on the jet centerline, and pressure fluctuations at $\phi=60^{\circ}$ and $d=240 r_{e}$ from the nozzle exit; - - - time delay for a rectilinear propagation at the ambient speed of sound. Time is normalized by $u_{e} /\left(2 r_{e}\right)$.

(a)

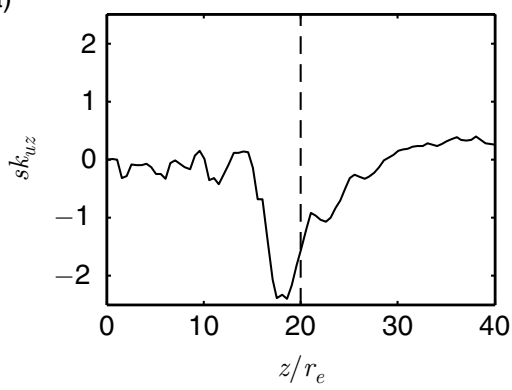

(b)

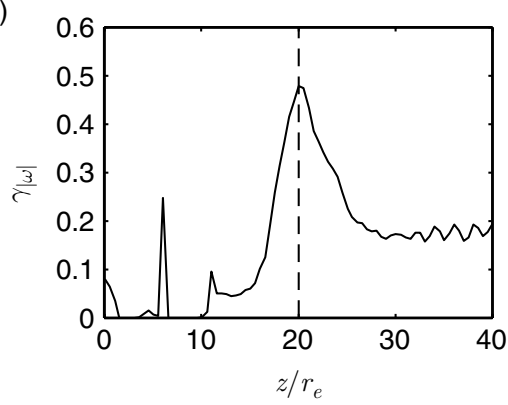

Figure 13: Centerline variations of (a) the skewness factor of axial velocity fluctuations and of (b) the intermittency factor of vorticity norm; - - end of the potential core.

a minimum equal to -2.4 at $z=18.5 r_{e}$. They indicate that large velocity deficits intermittently appear upstream of the end of the potential core.

The intermittency of the centerline turbulence is then investigated by evaluating the intermittency factor $\gamma_{|\omega|}$ defined as the time average of the function

$$
I(t)=\left\{\begin{array}{l}
1 \text { if }|\omega| \text { is lower than }<|\omega|>/ 2 \\
0 \text { otherwise }
\end{array}\right.
$$

as proposed in a previous study [27]. The value of $\gamma_{|\omega|}$ is expected to be close to 1 when the vorticity signal is very intermittent. The axial variations of $\gamma_{|\omega|}$ calculated from the jet centerline vorticity are presented in figure 13(b). Downstream of the nozzle exit, the 
intermittency factor is below 0.1, except at $z=6 r_{e}$ where a narrow peak is observed. It noticeably increases from $z=15 r_{e}$, and reaches a peak value of 0.47 at $z=20 r_{e}$ where the jet potential core closes. Farther downstream, the intermittency factor decreases, and values around 0.2 are obtained between $z=25 r_{e}$ and $z=40 r_{e}$. In the present supersonic jet, the vorticity signal is therefore strongly intermittent at the end of the potential core, in the same way as in subsonic jets [27].

In order to illustrate the flow properties at the end of the potential core, signals of $|\omega|$ and $u_{z}-u_{j}$ at $r=0$ and $z=z_{c}=20 r_{e}$ are provided in figures 14(a-b) using a time normalization by $u_{e} /\left(2 r_{e}\right)$. At certain times, for example at $t=233$ and $t=271$, sudden bursts of high intensity emerge in the vorticity signal. Simultaneously, negative peaks are found in the axial velocity signal, leading to velocity deficits reaching up to about $50 \%$ of $u_{j}$. Similar signal features are noted at the end of the potential core of subsonic jets [27]. They can be associated with the intermittent intrusion of low-velocity vortical structures in the jet core. These structures are then accelerated, which is assumed to generate noise in the downstream direction [27].

For the present jet, significant correlations are obtained in figure 10(c) between the centerline vorticity norm near $z=20 r_{e}$ and the far-field pressure fluctuations at $\phi=60^{\circ}$ for time delays around the acoustic propagation time. The pressure at $\phi=60^{\circ}$ and $d=240 r_{e}$ is thus shown in figure 14(c) as a function of time $t-\tau$ normalized by $u_{e} /\left(2 r_{e}\right)$, where $\tau$ is the time delay of maximum correlation between the pressure signal and the vorticity norm at the end of the potential core. Coincidences appear between the positive part of the acoustic signal, and the positive and negative peaks of $|\omega|$ and $u_{z}^{\prime}$, see at $t=262$ and $t=298$ for instance. This is most likely the reason for the positive and negative flow-noise correlations observed respectively in figures 14(a) and 14(c). Similar results are found in subsonic jets [27], which suggests the presence of the same noise generation mechanism around the end of the potential core. This contention is also supported by the fact that the noise spectra of subsonic and supersonic jets in the downstream direction fit to the same similarity spectrum, as demonstrated by Tam [43].

\subsection{Correlations with shear-layer flow quantities}

The correlations calculated between the signals of $u_{z}^{\prime}, u_{r}^{\prime},|\omega|$ and $\rho^{\prime}$ at $r=r_{j}$ in the shear layer and the pressure fluctuations at $\phi=60^{\circ}$ are considered. They are represented in figure 15 as functions of the axial position $z / r_{e}$ along $r=r_{j}$ and of the time delay $\tau$ normalized by $u_{e} /\left(2 r_{e}\right)$. High levels of correlations are found close to the time delay for a straight-line propagation at the ambient speed of sound. As noted previously for the correlations with centerline flow quantities in figure 10, they increase with the propagation distance. The correlation maps obtained for $d=180 r_{e}$ and at $d=240 r_{e}$ are also very similar.

Concerning the pressure at $d=240 r_{e}$, large negative values of correlations are observed with the axial velocity fluctuations in figure 15(a)(iiii) between $z=12 r_{e}$ to $z=36 r_{e}$ around the acoustic propagation time, with a peak located between the ends of the potential core and of the sonic core. Appreciable correlation levels appear with the radial normal stress $u_{r}^{\prime} u_{r}^{\prime}$ in figure 15(b)(iiii) for $5 r_{e} \leq z \leq 30 r_{e}$, and with the 
(a)

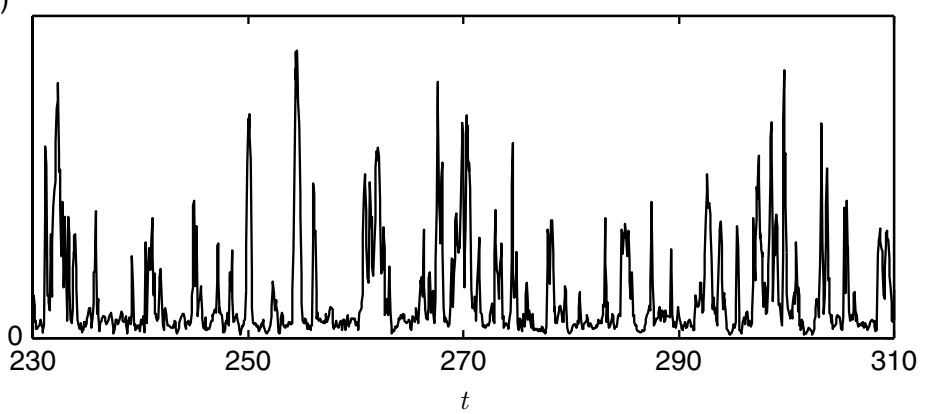

(b)

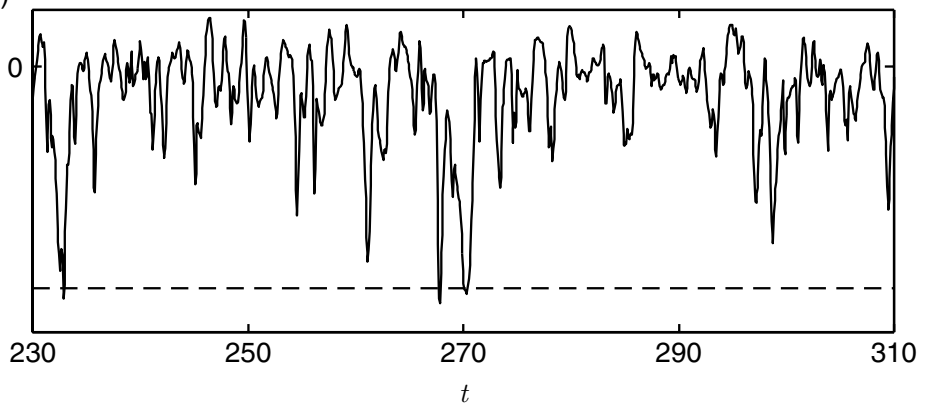

(c)

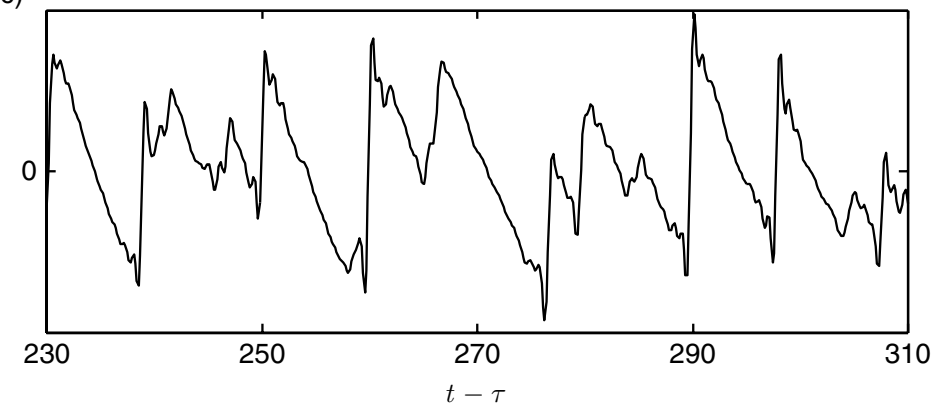

Figure 14: Time signals of (a) vorticity norm $|\omega|$ and (b) velocity $u_{z}-u_{j}$ at the end of the potential core on the jet axis, and (c) pressure fluctuations $p^{\prime}$ at $\phi=60^{\circ}$ and $d=240 r_{e}$ from the nozzle exit; - - - $u_{z}=u_{j} / 2, \tau$ time delay estimated from $C|\omega| p$ correlations. Time is normalized by $u_{e} /\left(2 r_{e}\right)$.

vorticity norm in figure 15 (c)(iiii) for $12 r_{e} \leq z \leq 30 r_{e}$. Positive correlation coefficients are finally noted with the density fluctuations in figure 15(d)(iiii) in the vicinity of the acoustic propagation time. 
(a)
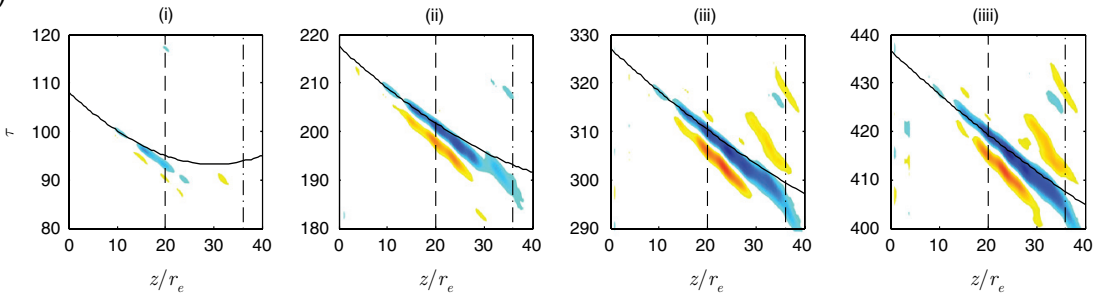

(b)
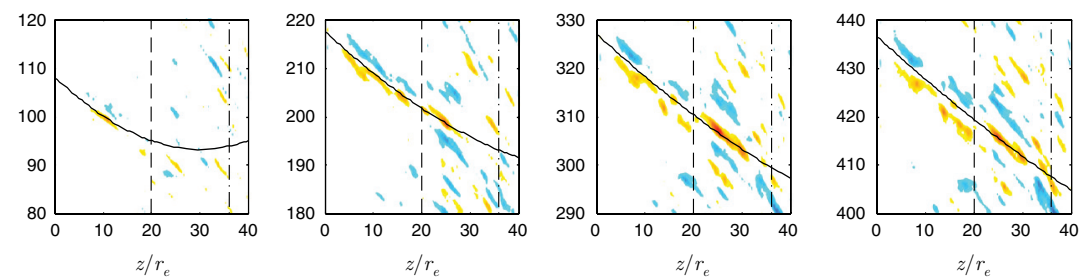

(c)
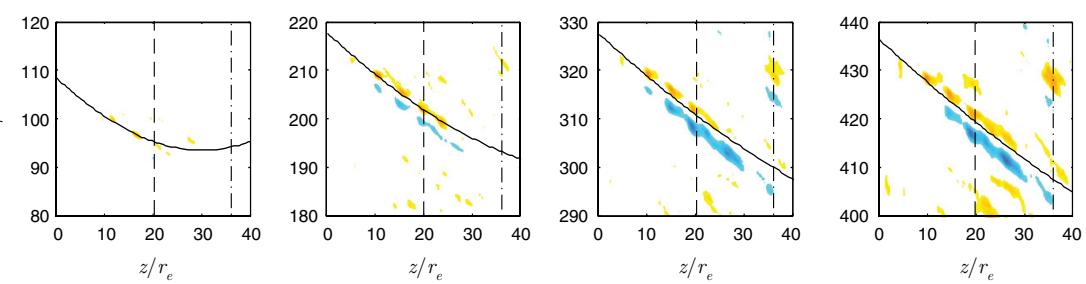

(d)
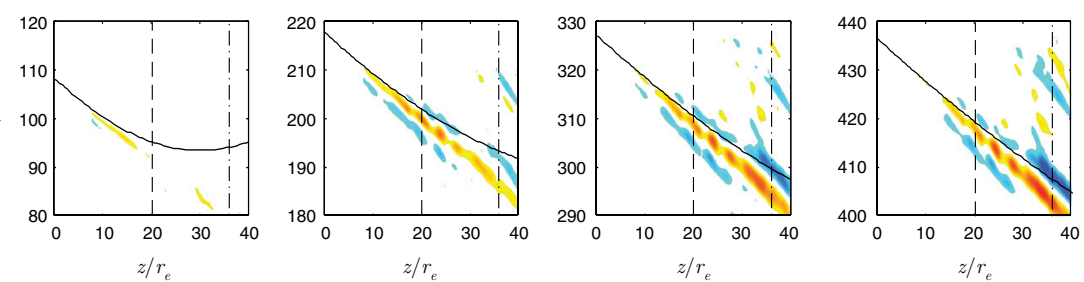

Figure 15: Normalized correlations between $p^{\prime}$ obtained for $\phi=60^{\circ}$ at (i) $d=60 r$, (ii) $d=120 r_{e}$, (iii) $d=180 r_{e}$ and (iiii) $d=240 r_{e}$ from the nozzle exit using non-linear wave extrapolation, and (a) $u_{z}^{\prime}$, (b) $u_{r}^{\prime} u_{r}^{\prime}$, (c) $|\omega|$ and (d) $\rho^{\prime}$ at $r=r_{j}$ (X-axis: position on the jet centerline, Y-axis: time delay normalized by $\left.u_{e} /\left(2 r_{e}\right)\right)$; - time delay for a rectilinear propagation at the ambient speed of sound, - - - end of the potential core, - - - end of the sonic core. The color scales range from -0.25 to 0.25 for ( $a$, d), from -0.10 to 0.10 for (b), and from -0.08 to 0.08 for (c).

The correlations in figures 15(iiii) can be compared with those in figures 10(iiii) evaluated between the centerline flow quantities and also the far-field pressure at $d=240 r_{e}$. For the axial velocity fluctuations, a great resemblance is seen. The 
correlation coefficients have the same sign and nearly the same magnitudes, and their maximum values are located for $z \geq z_{c}$ downstream of the jet potential core in both cases. For $z \leq z_{c}$, however, noticeable levels of correlations are found in the shear layer in figure 15(a)(iiii) along the trajectory of the acoustic propagation time, but not on the jet axis in figure 10(a)(iiii). The differences between the correlation maps are much larger for the other flow quantities. For $u_{r}^{\prime 2}$ and $|\omega|$, the correlations are for instance weaker in the shear layer than on the jet centerline. For the density fluctuations $\rho^{\prime}$, moreover, their sign clearly changes with the axial distance on the centerline in figure 10(d)(iiii), which is not the case in the shear layer in figure 15(d)(iiii).

The axial variations of the peak absolute values of the correlations computed between $u_{z}^{\prime}$ and $|\omega|$ at $r=r_{j}$ and $p^{\prime}$ at $d=60 r_{e}, 120 r_{e}, 180 r_{e}$ and $240 r_{e}$ are reported in figures 16(ab). In figure 16(a), the peak levels of $\left|C u_{z} p\right|$ increase with $d$, reaching maximum values of 0.12 at $z=15.5 r_{e}$ for $d=60 r_{e}, 0.19$ at $z=20 r_{e}$ for $d=120 r_{e}, 0.23$ at $z=24 r_{e}$ for $d=$ $180 r_{e}$, and 0.24 at $z=24.5 r_{e}$ for $d=240 r_{e}$. These results are in fair agreement with those obtained on the jet axis in figure 11(a). In figure 16(b), contrary to figure 16(a), the peak levels of $|C| \omega|p|$ vary neither with the propagation distance nor with the axial position along the shear layer. Furthermore, compared to figure 11(b), the correlation levels are lower, and no distinct peak emerges near the end of the potential core.

The correlations between the axial velocity fluctuations at $r=r_{j}$ and the pressure fluctuations at $\phi=60^{\circ}$ and $d=240 r_{e}$ are replotted in figure 17 along with the time delay predicted for a rectilinear propagation at the ambient speed of sound between the shear-layer and the far-field points. Large negative values are obtained between $z=12 r_{e}$ and $z=36 r_{e}$, for time delays very close to the propagation time delay for $z \leq 25 r_{e}$, but gradually deviating from the latter for $z \geq 25 r_{e}$. Therefore, only the correlations between $z=12 r_{e}$ and $z=25 r_{e}$ may be connected with noise generation.

As proposed in a previous work [27], a time delay $\tau_{\text {conv }}$ taking into account the convection of the turbulent structures in the axial direction is calculated. It is defined as

(a)

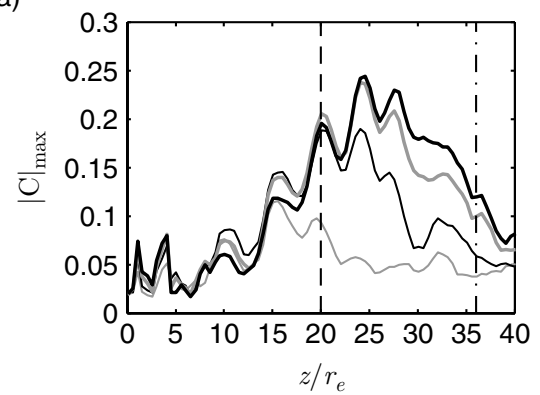

(b)

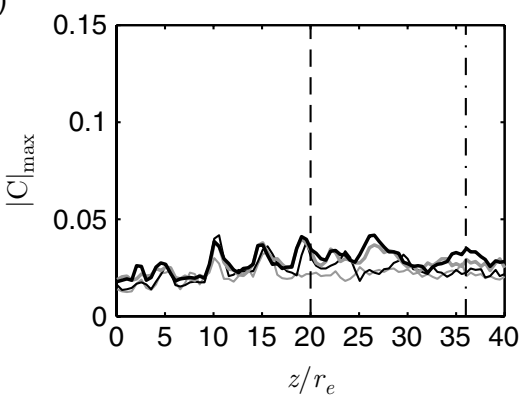

Figure 16: Axial variations of the peak absolute value of normalized correlations between (a) $u_{z}^{\prime}$ and (b) $|\omega|$ at $r=r_{j}$, and pressure fluctuations at $\phi=60^{\circ}$ and $\longrightarrow d=60 r_{e},-d=120 r_{e}, \quad d=180 r_{e}$ and $-d=240 r_{e}$ from the nozzle exit; - - - end of the potential core, -.- end of the sonic core. 


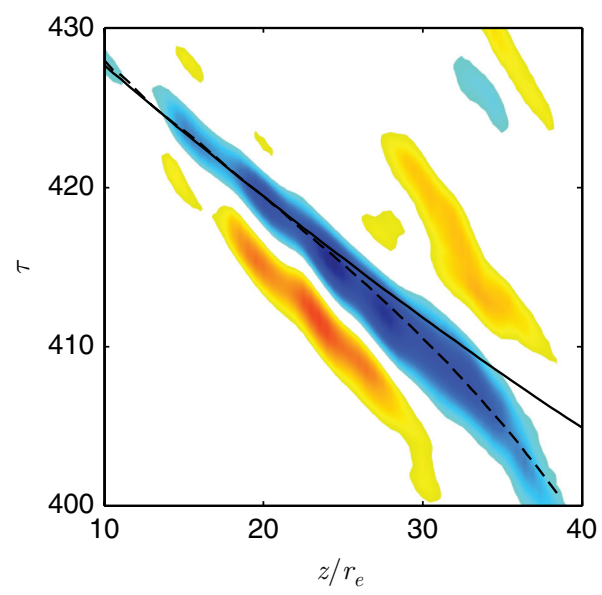

Figure 17: Normalized correlations between pressure fluctuations at $\phi=60^{\circ}$ and $d=240 r_{e}$ and axial velocity fluctuations at $r=r_{j} ;-$ time delay for a propagation at the speed of sound, - - - time delay $\tau_{\text {conv }}$ based on the convection velocity. Time is normalized by $u_{e} /\left(2 r_{e}\right)$. The color scale ranges from -0.25 to 0.25 .

$$
\tau_{\text {conv }}(z)=\tau_{a c}\left(z_{c}\right)+\int_{z}^{z_{c}} \frac{d z}{u_{c}(z)}
$$

where $\tau_{a c}\left(z_{c}\right)$ is the acoustic propagation time estimated for $z=z_{c}=20 r_{e}$ and $r=r_{j}$, and $u_{c}$ is the convection velocity at $r=r_{j}$ shown in figure 4(a), and is displayed in figure 17. The spot of strong negative correlations is aligned with $\tau_{\text {conv }}$, which suggests that the correlations downstream of $z=25 r_{e}$ are associated with the convection of noise sources.

It can be noted that the strong correlations between $z=12 r_{e}$ and $z=25 r_{e}$ are found at time delays agreeing with both the propagation and the convection time delays, which coincide with each other here. As the sign of the correlations do not change in this region, they may be linked to the same noise generation mechanism. The mechanism thus spreads over a large axial extent, and is related to the supersonic convection of turbulent structures. This description corresponds fairly well to that done by Ffowcs Williams \& Maidanik [44] for the Mach wave mechanism.

\subsection{Correlations with density fluctuations}

The correlations maps of figures 10(d)(iiii) and 15(d)(iiii), calculated between the pressure at $d=240 r_{e}$ and the density at $r=0$ and at $r=r_{j}$, respectively, are shown side by side in figures $18(\mathrm{a}-\mathrm{b})$. The time delay for a propagation at the speed of sound, and the time delay $\tau_{\text {conv }}$ estimated, as defined above, from the convection velocity at 
(a)

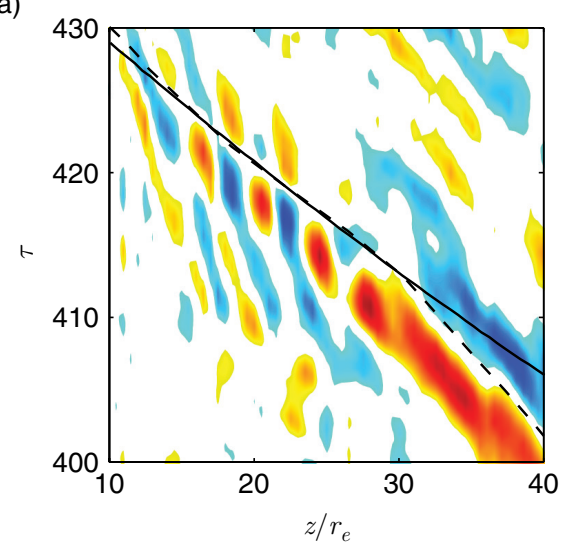

(b)

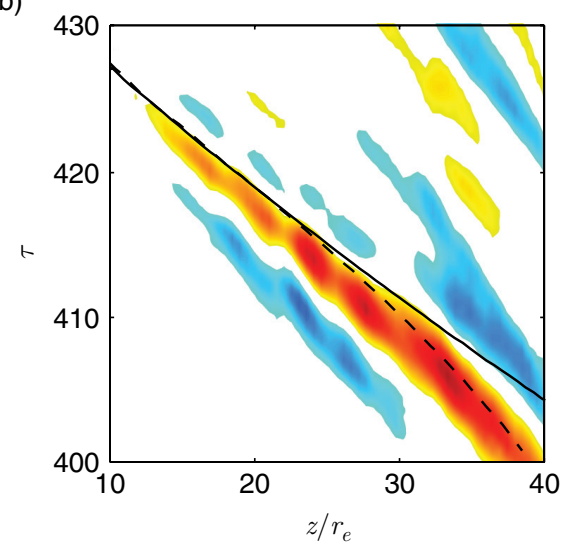

Figure 18: Normalized correlations between pressure fluctuations at $\phi=60^{\circ}$ and $d=240 r_{e}$ and density fluctuations at (a) $r=0$ and (b) $r=r_{j}$; time delay for a rectilinear propagation at the ambient speed of sound, - - - time delay $\tau_{\text {conv }}$ based on the convection velocity. Time is normalized by $u_{e} /\left(2 r_{e}\right)$. The color scale ranges from -0.25 to 0.25 .

$r=0$ and at $r=r_{j}$, are also displayed. They are very similar to each other from $z=12 r_{e}$ up to $z=30 r_{e}$ in figure 18(a), and from $z=12$ up to $z=25 r_{e}$ in figure 18(b). In both figures, significant correlation levels are found for time delays close the acoustic propagation time. However, the correlation sign changes periodically between $z=12 r_{e}$ and $z=25 r_{e}$ in figure 18(a) for the centerline density fluctuations, whereas it remains positive in figure 18(b) for the shear-layer density fluctuations. In the first case, red and blue stripes of positive and negative correlations alternately appear.

The correlations obtained with the centerline density fluctuations are represented again in figures 19 (a-b) in order to get more information on the alternating stripes of positive and negative correlations. As the jet is overexpanded, it is first natural to compare the quasi-periodic variations of the correlations in the axial direction with the oscillations of the centerline mean static pressure $\langle p\rangle$, reported in figure 2(a). The positions of the maxima and minima of $\langle p\rangle$ are thus indicated in figure 19(a). Near the acoustic propagation line, between $z=12 r_{e}$ and $z=25 r_{e}$, that is between the 3rd and the 5th shock cells, they fall in the regions where the correlation values are zero. This reveals that the successive bands of correlations are related to the shock-cell structure. Moreover, the correlations are positive when the flow is compressed, and negative when it is expanded.

In order to discuss the direction of the correlation stripes, which seems to be linked neither to the acoustic time nor to the convection time, it is recalled that screech noise components were identified in the far-field spectra of the present jet in a previous study [13] with a fundamental frequency at a Strouhal number of $S t_{\text {screech }}=0.08$, see the 
(a)

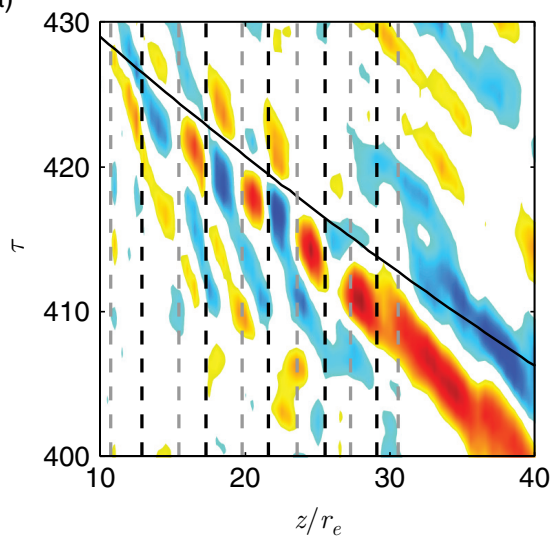

(b)

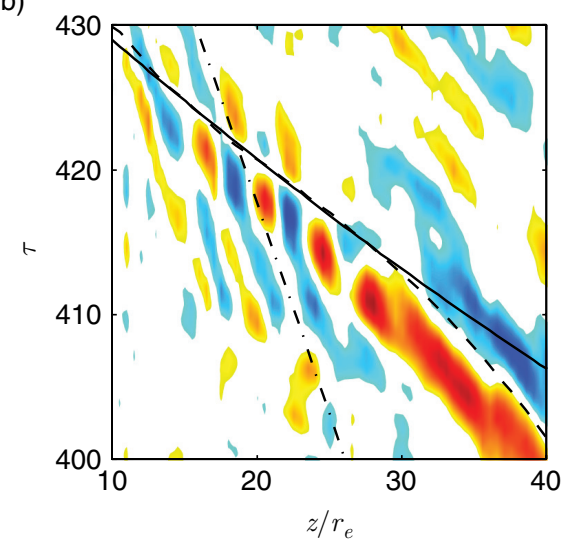

Figure 19: Normalized correlations between pressure fluctuations at $\phi=60^{\circ}$ and $d=240 r_{e}$ and density fluctuations at $r=0, \longrightarrow$ time delay for a rectilinear propagation at the ambient speed of sound; (a) positions of the - - - maximum and --- minimum values of mean static pressure on the jet centerline; (b) - - - time delay $\tau_{\text {conv }}$ based on the convection velocity, and -.-.- time delay $\tau_{\text {screech }}$ based on the screech frequency. Time is normalized by $u_{e} /\left(2 r_{e}\right)$. The color scale ranges from -0.25 to 0.25 .

upstream-propagating waves in figure 1. A time delay $\tau_{\text {screech }}$ based on the screech time period $\tau_{\text {screech }}$ and the local shock-cell length $L_{\text {shock }}$ is then built as

$$
\tau_{\text {screech }}(z)=\tau_{a c}\left(z_{0}\right)+\int_{\mathrm{z}}^{z_{0}} \frac{d z}{u_{\text {screech }}(z)}
$$

where $z_{0}$ is an arbitrary position on the jet axis, $\tau_{a c}\left(z_{0}\right)$ is the acoustic time delay for $z=z_{0}$, and $u_{\text {screech }}=L_{\text {shock }} / T_{\text {screech }}$ is the average velocity needed to move a shock cell distance during the screech time period. The screech time screech obtained for $z_{0}=18.5 r_{e}$, where the shock-cell length estimated from figure $2(\mathrm{a})$ is $\mathrm{L}_{\text {shock }}=4.3 r_{e}$, is depicted in figure 19(b). The trajectory followed by $\tau_{\text {screech }}$ agrees well with the direction of the correlation stripes, which suggests that they are related to the screech noise. This may be due to the fact that in screeching jets, shocks oscillate at the screech frequency, as observed experimentally by Panda [45] and André et al. [46] for instance.

\section{CONCLUSION}

In this paper, the properties of the noise emitted by an overexpanded round jet at a Mach number of 3.3 and a Reynolds number of $10^{5}$ are investigated. Non-linear propagation effects are first shown to be significant up to a distance of about 240 radii from the jet nozzle exit, in particular at the peak directivity radiation angle. They spectacularly distort the acoustic waves, leading to a series of $N$-shaped waves in the pressure signals, 
and to weaker mid-frequency components and stronger high-frequency components in the spectra. The far-field pressure fluctuations are then found to correlate with turbulent quantities, including axial velocity fluctuations, vorticity norm and density fluctuations, located on the jet centerline and in the shear layer, providing information on noise generation mechanisms. High levels of correlation are found with the flow around the end of the jet potential core. They are attributed to the intermittent intrusion of lowspeed vortical structures in the core, as is the case in subsonic jets. Appreciable correlations are also obtained with fluctuations in the jet shear layer over a large axial distance. They may be related to the supersonic convection of turbulent structures. Finally, alternating stripes of positive and negative correlations are observed with the centerline density fluctuations between the 3rd and the 5th shock cells. They appear to be linked to the shock motions at the screech tone frequency.

\section{ACKNOWLEDGMENTS}

The work presented in this paper was performed during the postdoctoral training of the first author sponsored by the CNES (Centre National d'Etudes Spatiales) with Hadrien Lambaré as supervisor. It was granted access to the HPC resources of the Institut du Développement et des Ressources en Informatique Scientifique (IDRIS) under the allocation 2012-020204 made by GENCI (Grand Equipement National de Calcul Intensif).

\section{REFERENCES}

[1] Laufer, J., Schlinker, R. and Kaplan, R.E., Experiments on supersonic jet noise, AIAA Journal, 1976, 14, 489-504.

[2] Tam, C., Supersonic jet noise, Annual Review of Fluid Mechanics, 1995, 27, 17-43.

[3] Freund, J.B., Noise sources in a low-Reynolds-number turbulent jet at Mach 0.9, Journal Fluid Mechanics, 2001, 438, 277-305.

[4] Bogey, C., Bailly, C. and Juvé, D., Noise investigation of a high subsonic, moderate Reynolds number jet using a compressible LES, Theoretical and Computational Fluid Dynamics, 2003, 16(4), 273-297.

[5] Shur, M.L., Spalart, P.R., Strelets, M.Kh. and Travin, A.K, Towards the prediction of noise from jet engines, International Journal of Heat and Fluid Flow, 2003, 24(4), 551-561.

[6] Bodony D.J. and Lele, S.K., On using large-eddy simulation for the prediction of noise from cold and heated turbulent jets, Physics of Fluids, 2005, 17(8), 085103.

[7] Bogey, C. and Bailly, C., Influence of nozzle-exit boundary layer conditions on the flow and acoustic fields of initially laminar jets, Journal Fluid Mechanics, 2010, 663, 507-538.

[8] Bogey, C., Marsden, O. and Bailly, C., Large-eddy simulation of the flow and acoustic fields of a Reynolds number $10^{5}$ subsonic jet with tripped exit boundary layers, Physics of Fluids, 2011, 23, 035105.

[9] Bogey, C., Marsden, O. and Bailly, C., Influence of initial turbulence level on the flow and sound fields of a subsonic jet at a diameter-based Reynolds number of $10^{5}$, Journal Fluid Mechanics, 2012, 701, 352-385. 
[10] Freund J.B, Lele, S.K. and Moin, P., Numerical simulation of a Mach 1.92 turbulent jet and its sound field, AIAA Journal, 2000, 38(11), 2023-2031.

[11] Berland, J., Bogey, C. and Bailly, C., Numerical Study of screech generation in a planar supersonic jet, Physics of Fluids, 2007, 19(7), 075105.

[12] Shur, M.L., Spalart, P.R. and Strelets, M.Kh., Noise prediction for underexpanded jets in static and flight conditions, AIAA Journal, 2011, 49(9), 2000-2017.

[13] de Cacqueray, N., Bogey, C. and Bailly, C., Investigation of a high-Mach-number overexpanded jet using large-eddy simulation, AIAA Journal, 2011, 49(10), 2171-2182.

[14] Mendez, S., Shoeybi, M., Sharma, A., Ham, F. E., Lele, S. K. and Moin, P., LES of perfectlyexpanded supersonic jets using an unstructured solver, AIAA Journal, 2012, 50(5), 1103-1118.

[15] Liu, J., Kailasanath, K., Boris, J.P., Heeb, N., Munday, D. and Gutmar, E, Effect of the Initial Turbulence Level on an Underexpanded Supersonic Jet, AIAA Journal, 2013, 51(3),741-745.

[16] Nichols, J.W., Lele, S.K., Ham, F.E., Martens, S. and Spyropoulos, J.T., Crackle noise in heated supersonic jets, Journal of Engineering for Gas Turbines and Power, 2013, 135, 051202.

[17] Morfey, C.L. and Howell, G.P., Nonlinear propagation of aircraft noise in the atmosphere, AIAA Journal, 1980, 19(8), 986-992.

[18] Gee, K.L., Sparrow, V.W., James, M.M., Downing, J.M., Hobbs, C.M., Gabrielson, T.B. and Atchley, A.A., The role of nonlinear effects in the propagation of noise from high-power aircraft, Journal of Acoustical Society of America, 2008, 123(6), 4082-4093.

[19] Viswanathan K., Distributions of noise sources in heated and cold jets: are they different?, International Journal of Aeroacoustics, 2006, 9(4\&5), 589-626.

[20] Petitjean, B.P., Morris, P.J. and McLaughlin, D.K., On the nonlinear propagation of shockassociated jet noise, AIAA Paper 2005-2930, 2005.

[21] Petitjean, B.P., Viswanathan K. and McLaughlin, D.K., Acoustic pressure waveforms measured in high speed jet noise experiencing nonlinear propagation, International Journal of Aeroacoustics, 2006, 5(2), 193-215.

[22] Saxena, S., Morris, P.J. and Viswanathan, K., Algorithm for the nonlinear propagation of broadband jet noise, AIAA Journal, 2009, 47(1), 186-194.

[23] Baars, W., Tinney, C.E. and Wochner, M.S., Nonlinear noise propagation from a fully expanded Mach 3 jet, AIAA Paper 2012-1177, 2012.

[24] Baars, W. and Tinney, C.E., Shock-structures in the acoustic field of a Mach 3 jet with crackle, Journal of Sound and Vibration, 2014, 333, 2539-2553.

[25] Mora, P., Heeb, N., Kastner, J., Gutmark, E.J. and Kailasanath, K., Impact of heat on the pressure skewness and kurtosis in supersonic jets, AIAA Journal, 2014, 52(4), 777-787.

[26] Panda, J., Experimental investigation of turbulent density fluctuations and noise generation from heated jets, Journal of Fluid Mechanics, 2007, 591, 349-385. 
[27] Bogey, C. and Bailly, C., An analysis of the correlations between the turbulent flow and the sound pressure field of subsonic jets, Journal of Fluid Mechanics, 2007, 583, 71-97.

[28] Bogey, C., Barré, S., Juvé, D. and Bailly, C., Simulation of a hot coaxial jet: direct noise prediction and flow-acoustics correlations, Physics of Fluids, 2009, 21, 035105.

[29] Grizzi, S. and Camussi, R., Wavelet analysis of near-field pressure fluctuations generated by a subsonic jet, Journal of Fluid Mechanics, 2012, 698, 93-124.

[30] Henning, A., Koop, L. and Schröder, A., Causality correlation analysis on a cold jet by means of simultaneous Particle Image Velocimetry and microphone measurements, Journal of Sound and Vibration, 2013, 332, 3148-3162.

[31] Panda, J. and Seasholtz, R.G., Experimental investigation of density fluctuations in highspeed jets and correlation with generated noise, Journal of Fluid Mechanics, 2002, 450 97-130.

[32] Panda, J., Seasholtz, R.G. and Elam, K.A., Investigation of noise sources in highspeed jets via correlation measurements, Journal of Fluid Mechanics, 2005, 537, 349-385.

[33] Papamoschou, D., Morris, P.J. and McLaughlin, D.K., Beamformed flow-acoustic correlations in a supersonic jet, AIAA Journal, 2010, 48(10), 2445-2453.

[34] Veltin, J., Day, B.J. and McLaughlin, D.K., Correlation of flowfield and acoustic field measurements in high-speed jets, AIAA Journal, 2011, 49(1), 150-163.

[35] Varnier, J. and Gély, D., Caractérisation aérodynamique et acoustique d'un jet fortement supersonique en présence d'un obstacle plan, ONERA RT-112/3643, 1998 (not published).

[36] Bogey, C. and Bailly, C., A family of low dispersive and low dissipative explicit schemes for flow and noise computations, Journal of Computational Physics, 2004, 194(1), 194-214.

[37] Berland, J., Bogey, C., Marsden, O. and Bailly, C., High-order, low dispersive and low dissipative explicit schemes for multi-scale and boundary problems, Journal of Computational Physics, 2007, 224(2), 637-662.

[38] Bogey, C., de Cacqueray, N. and Bailly, C., A shock capturing methodology based on adaptative spatial filtering for high-order non-linear computations, Journal of Computational Physics, 2009, 228(5), 1447-1465.

[39] Mohseni, K. and Colonius, T., Numerical treatment of polar coordonate singularities, Journal of Computational Physics, 2000, 157(2), 787-795.

[40] Bogey, C., de Cacqueray, N. and Bailly, C., Finite differences for coarse azimuthal discretization and for reduction of effective resolution near origin of cylindrical flow equations, Journal of Computational Physics, 2011, 230(3), 1134-1146.

[41] Bogey, C. and Bailly, C. Turbulence and energy budget in a self-preserving round jet: direct evaluation using large-eddy simulation, Journal of Fluid Mechanics, 2009, 627, 129-160. 
[42] Bogey, C. and Bailly, C., Three-dimensional non-reflective boundary conditions for acoustic simulations: far field formulation and validation test cases, Acta Acustica united with Acustica, 2002, 8(4), 463-471.

[43] Tam, C.K.W., Jet noise: Since 1952, The sources of jet noise: experimental evidence, Theoretical and Computational Fluid Dynamics, 1998, 10, 393-405.

[44] Ffowcs Williams, J.E. and Maidanik, G., The Mach wave field radiated by supersonic turbulent shear flows, Journal of Fluid Mechanics, 1965, 21, 641-657.

[45] Panda, J., Shock oscillation in underexpanded screeching jets, Journal of Fluid Mechanics, 1998, 363, 173-198.

[46] André, B., Castelain, T. and Bailly, C., A shock tracking procedure for studying screech induced oscillations, AIAA Journal, 2011, 49(7), 1563-1566. 